\title{
Critical revision of the role of surgery for treatment of primary brain b cell lymphoma in an immunocompetent patient: a case report and literature review
}

\begin{abstract}
Primary Central Nervous System Lymphoma (PCNSL) are malignant tumors extremely rare in immunocompetent patients. They represent $2-3 \%$ of all cerebral tumors. They are frequently located in the supra-tentorial space and in $60-70 \%$ of cases they consist in single lesions. The most common presenting symptoms are hemi paresis, ache, epileptic crises and ataxia. In this article we present the case of a 76 year-old woman admitted for seizures attack and progressive coma state. A preoperative CT scan with contrast enhancement showed a left frontal nodular lesion of 29x16mm, with irregulars enhancement and surrounded by peripheral edema. No alteration of haematological exams was noticed. The patient underwent surgery for with total excision of the lesion. Histological examination was suggestive of non-Hodgkin B cells lymphoma (CD20+, CD 30-). Patient improved after surgery and she was discharged ten days after surgery, with the indication to perform hematologic consult. No recurrences were noticed at 20months follow-up. Although surgery is not routinely recommended for the treatment of PCNSL, we report a case of B-cells lymphoma in which surgical resection, followed by chemotherapy and radiation therapy, resulted in a successful treatment. A critical review of the role of the surgery in consideration of technical advances in neurosurgery was done.
\end{abstract}

Volume 8 Issue 2 - 2018

Rizzi Gaetano,' Berardi Arturo,' Bozzini

Vincenzo,' Donnarumma Pasquale,' Rauseo

Michela, ${ }^{2}$ Merlicco Gaetano'

'Department of Neurosurgery, Azienda Ospedaliero-

Universitaria OO-RR Foggia, Italy

${ }^{2}$ Department of Anaesthesia and Intensive Care Unit Azienda

Ospedaliero-Universitaria OO-RR Foggia, Italy

Correspondence: Gaetano Rizzi, Designation, Neurosurgeon, University:Azienda Ospedaliero-Universitaria,"OO-RR” of Foggia, Address Viale Pinto, Italy, Tel +39088I733742, Email gaetanorizzi6@gmail.com

Received: December 08, 2017 | Published: March 21, 2018

\section{Introduction}

Primary Central Nervous System Lymphoma (PCNSL) consists in diffuse large $\mathrm{B}$ cell non-Hodgkin lymphoma usually confining to the CNS. PCNSL is well known to be a form of extra nodal, highgrade non-Hodgkin B-cell neoplasm. It can originate in the brain, leptomeninges, spinal cord, or eyes; usually it remains confined to the CNS, rarely it spreads outside the nervous system. It was previously considered as a rare tumor accounting for less than $2 \%$ of cerebral neoplasms, PCNSL is now being found with increasing frequency in immunocompetent patients. Although the cells of origin are lymphocytes (usually large cell or immunoblastic type), PCNSL should be considered a brain tumor, because its therapeutic challenges resemble those of other brain tumors. In particular, drug delivery is impaired by the blood-brain barrier, and cerebral toxicity limits the use of available treatment strategies. Most PCNSLs (about 90\%) are diffuse large B-cell lymphomas (DLBCLs); the remaining 10\% are poorly characterized low-grade lymphomas, Burkitt lymphomas, and T-cell lymphomas. ${ }^{1}$ Primary symptoms may be the sum of local mass effect due to raised intracranial pressure, or ocular involvement, or from focal deposits on cranial or spinal nerve roots. PCNSL incidence has risen steadily since the end of the $20^{\text {th }}$ century. Incidence in immunocompetent patients is approximately 51 cases per 10,000,000 per year. Among immunocompetent patients with PCNSL, males outnumber females by approximately 2:1 The median age of immunocompetent patients with PCNSL is 55 years. $^{2}$

\section{Case report}

A 76 year-old female admitted with generalized epileptic status and sudden coma. A brain CT scan documented a nodular lesion in the right front lobe of the size of $29 \times 16 \mathrm{~mm}$, with inhomogeneous enhancement after intravenous contrast and peri-lesional edema (Figure 1). She underwent surgery consisting in a total removal of the tumor. The histological examination showed a "non-Hodgkin large B-cell lymphoma (CD20+, CD 30-)" (Figures 2-4). Post-operative treatment consisted in seven days mannitol (50gr, three times a day) and dexamethasone $(10 \mathrm{mg}$, twice daily). A post-operative CT scan showed the total excision of the tumor. After histological examination, the patient underwent hematologic consult and treatment according to the R-MPV protocol. She was discharged ten days after surgery in good conditions. In April 2016 a CT scan showed a subcortical hypodensity in right frontal lobe, without any new hypo-density zones. From June to July 2016 the patients developed clinical symptom of neurotoxicity with a sever short-term memory loss, incontinence, disabling dementia, which had a negative impact on patients' quality of life. The patient died in August 2016, 24 months after the diagnosis (Figure 5).

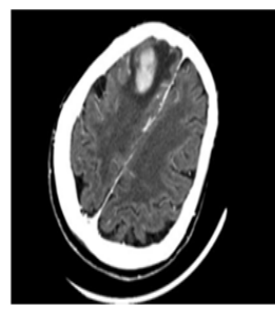

Figure I Preoperative CT scan after contrast enhancement document a nodular lesion in the right front lobe of the size of $29 \times 16 \mathrm{~mm}$, with inhomogeneous enhancement and peri-lesional edema after intravenous contra. 


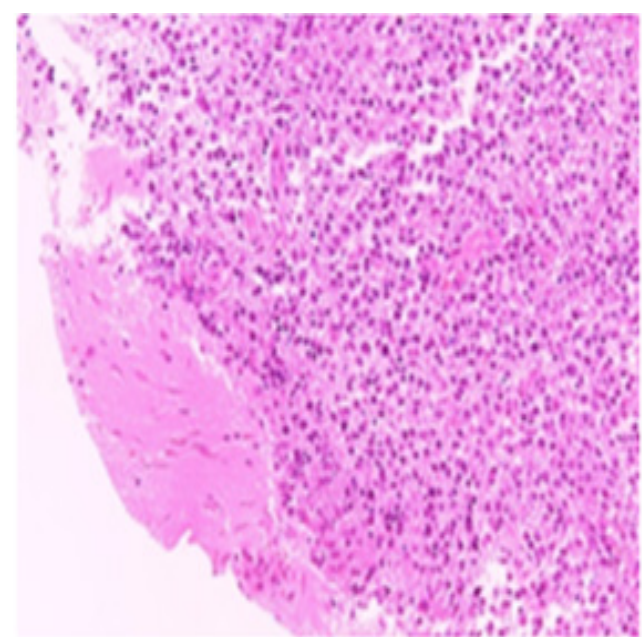

Figure 2 Diffuse proliferation of large lymphoid cells, sharply demarcated from surrounding cerebral parenchyma (H\&E, 200x).

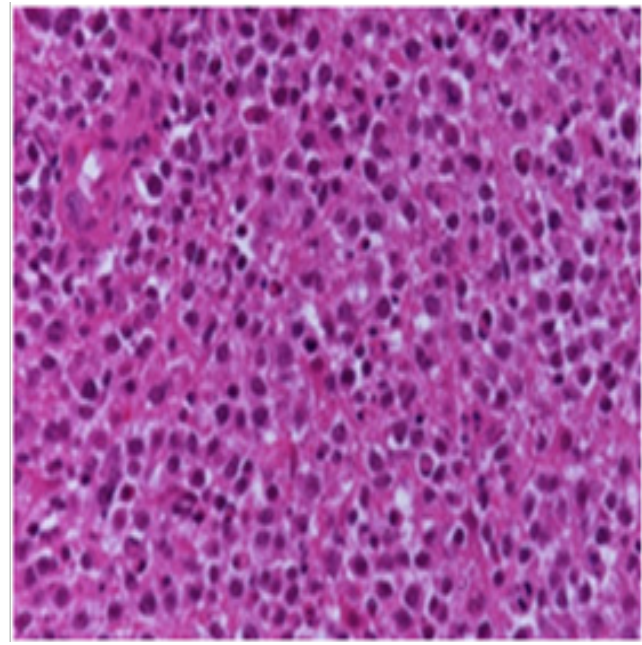

Figure 3 With high power magnification, tumor cells show round to ova nuclei with prominent nucleoli and several mitoses (H\&E, 400x).

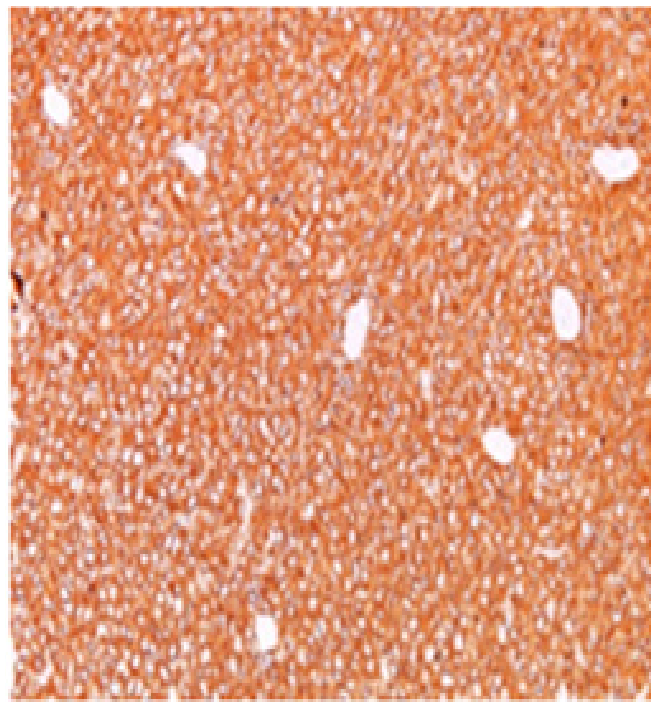

Figure 4 CD20 staining demonstrating B-cell phenotype of neoplastic cells (IHC, 200x).

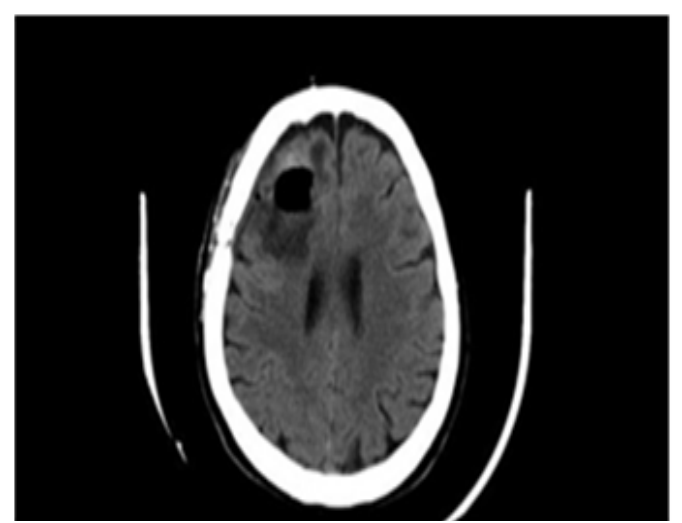

Figure 5 Post-operative CT scan shows the total excision of the tumor.

\section{Discussion}

Althought several studies suggest with reasonable basis the following sequence for the treatment of primary intracerebral lymphoma: stereotactic biopsy sampling, chemotherapy with a methotrexate- and anthracycline-based regimen, followed by cranial irradiation, ${ }^{3}$ the possible application of surgery to the treatment of PCNSL still remains controversial. With the development of neurosurgical techniques, in particular of the neuro navigation systems and functional neurosurgery such as awake-surgery, nowadays neurosurgeon can reach critical and deep areas with major safety. PCNSLs represent $0.7-1.7 \%$ of all malignant brain tumors, occurring in more than $50 \%$ of cases in supra-tentorial areas. ${ }^{4,5}$ They are characterized by high aggressiveness and rapid growth. Symptoms appear in 3-6months and can be aspecific, including headache, cognitive deficits, and psychomotor slowing. ${ }^{6,7}$ MRI scan is the gold standard for diagnosis, which aim to discriminate lymphoma from other brain tumors. However, definitive diagnosis requires brain biopsyor cerebrospinal fluid analysis. Although the use of surgery for PCNSL patients is still under debate, ${ }^{8-10}$ the present case report suggests that surgical resections are useful for the management of PCNSL, particularly when they are used in combination with chemotherapy and radiotherapy regimens. Specimens analysis confirmed the diagnosis of PCNSL, and provided useful information for the management of the disease. In this case the patient arrived to our observation in poor neurologic conditions due to continuous epileptic status, with a single occupied lesion and increased intracranial pressure. After surgery, with the removal of epileptogenic area, the symptoms of the patient were significantly improved without complications. Differential diagnosis includes metastasis, glioma and infections, whereas in rare cases PCNSLs have signs of demyelinating disease. ${ }^{11}$ Over the last ten years, several studies did not recommend surgical excision as a primary treatment since the mass present diffuse infiltration and the disease may be controlled by chemotherapy and radiotherapy. ${ }^{1,3,12}$ Moreover these tumors are often cited in deep locations and therefore the risk of post-operative neurologic complications is increased. Surgical intervention is restricted to stereotactic biopsy, which is required to confirm the diagnosis. ${ }^{13}$ According to several Authors, tumor removal should be considered to treat lesions amenable to resection, especially in cases of single lesions. ${ }^{14}$ In 1974, Henry and colleagues ${ }^{15}$ reported survival of 3.3 months in 15 cases managed with supportive care alone and 4.6 months in 28 cases managed after surgery alone, but 15.2 months in 21 cases treated with radiotherapy, with or without surgery. De Angelis and colleagues $^{16}$ observed no complication in 19 cases managed with 
stereotactic biopsies, whereas 4 of 10 patients who had had a complete resection suffered a severe postoperative deficit, indicating an increased surgical risk in this patient population. Bellinzona et al., ${ }^{17}$ in a recent retrospective study ${ }^{17}$ of 32 patients also questioned the value of surgical resection, although the authors essentially acknowledged that their study was inconclusive. In a retrospective analysis of 248 patients, the one-year survival rate was higher in patients who underwent complete excision than in those who underwent stereotactic biopsy and although this result was not statistically significant, it could imply therapeutic benefit. ${ }^{3-18}$ The most recent study published examining the possible benefit of surgical resection in PCNSL is a 10 -year retrospective series of 27 patients, with 12 undergoing total tumor resection, with all patients receiving high dose methotrexate. Patients who received complete resection had a significantly longer overall survival compared to partial resection or biopsy only patients. ${ }^{19}$ Although this study was limited by its small sample size, retrospective nature, and lack of reported complications, it suggests that a potential benefit for gross total resection in PCNSL exists. Previous studies supported that the surgery may no longer be enough to assess its curative role, because the technical advances in neurosurgery and the amelioration of adjuvant treatments have contributed to the efficiency and safety of surgical resections. Therefore, future studies should be conducted in order to evaluate extent of tumor removal in PCNSL patients. Diffuse disease and inaccessible location are additional arguments against the role of the surgery in treatment of PCNSL. PCNSL can be found in eloquent or difficult to access areas such as deep brain structures, making resective surgery in cases difficult and considerably morbid. Intraocular or leptomeningeal dissemination, and cyto reduction is thought of as futile in this scenario..$^{20}$ Further, there is a concern that surgery might create dissemination of tumor cells in to the subarachnoid space. ${ }^{21}$ In addition, PCNSL can respond rapidly to corticosteroids and chemotherapy, with eventual resolution of mass effect and neurological symptoms, foregoing the need for debulking surgery. Most surgeons would recommend to wait for a period after surgery to allow for wound healing, and there is a concern that minor delays in treatment worsen outcomes for PCNSL. ${ }^{22}$ Due to these considerations, surgical resection with the goal of cyto reduction has not been the primary surgical strategy in the management of this disease. Radiation and chemotherapy has an important role in improving the survival rates of PCNSL patients, particularly when chemotherapy agents are administered prior to radiation therapy. ${ }^{23}$ Several chemotherapic protocols are nowadays used to treat these patients. ${ }^{24}$ High-dose methotrexate (dose $>1 \mathrm{~g} / \mathrm{m}^{2}$ ) is the most effective medication for the treatment of PCNSL ${ }^{25}$ since it is able to determine response rates between 52 and $88 \%$ in single dose and between 70 and $94 \%$ when used in combination. So far, it has not been shown that an additional medication could add benefit to high-dose methotrexate used in chemotherapy. In clinical practice, HD-MTX, alone or in combination, should be the treatment of choice in patients with PCNSL. ${ }^{26}$ A previous study demonstrated that five cycles of HD-MTX $\left(3.5 \mathrm{~g} / \mathrm{m}^{2}\right)$ and procarbazine $\left(100 \mathrm{mg} / \mathrm{m}^{2} /\right.$ day $)$ is beneficial for patients with a $90 \%$ objectiveresponse rate and mediansurvival of 60 months. ${ }^{9}$ Because of their low penetration through the BBB ( brain blood barrier), the two most active drugs in the treatment of non-Hodgkin's lymphoma, doxorubicin and cyclophosphamide, are associated with unsatisfactory results in the treatment of PCNSL. ${ }^{27}$ Although at the beginning this treatment seems to success, most of the patients treated with the CHOP or MACOP-B experienced a during the chemotherapy treatment. ${ }^{28}$ Other combination treatments included high-dose MTX chemotherapy, temozolomide and Rituximab.,29 Rituximab, a chimeric monoclonal antibody directed against the CD20 Antigen specific B lymphocytes, is actually object of debate. ${ }^{8}$ Regarding radiation therapy, the whole brain irradiation with 40-50Gy followed by localized irradiation of $60 \mathrm{~Gy}$ on regions of edema is recommended as the best regimen..$^{30}$ Our patient received 7 cycles of chemotherapy (PMV), and two cycles of ARACYTINE for further consolidation: $3000 \mathrm{mg} / \mathrm{m}^{2}$ or $584 \mathrm{mg}$ per 24 hours, followed by whole brain radiation therapy $(23.4 \mathrm{~Gy})$ and additional radiotherapy until reaching 40Gy in one month. No recurrence was noticed at 22months follow-up (Figure 6). Previous studies indicate that PCNSL patients survive for three to six months with no treatment. ${ }^{31,32}$ Although comprehensive therapy improves the progression freeand the overall survival (OS) rates, when compared with untreated patients, the five years survival rate of patients that receive treatment remains 20$25 \%{ }^{33}$ In addition, $35-60 \%$ of patients experience ectopic recurrence within two years of diagnosis. Patients that experience the recurrence of PCNSL demonstrate an OS of 8-18months. Data show there is no standard treatment for recurrent PCNSL. ${ }^{34}$ In the present case report, subsequent 22 months of follow-up, the patient had a normal life and experienced no additional recurrence.

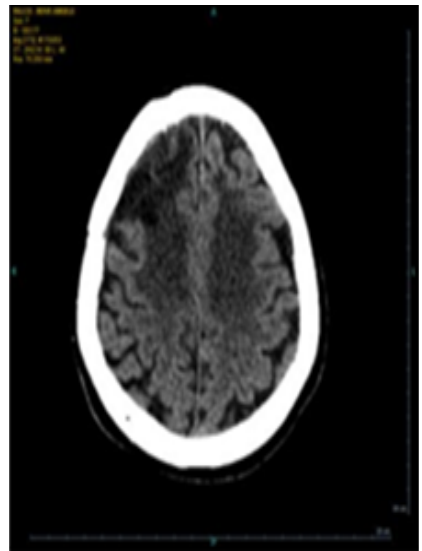

Figure 6 Follow-up 28/04/2016, subcortical hypodensity in right frontal lobe, in absence of new other hypodensity zones.

\section{Conclusion}

Non-Hodgkin large B cell lymphoma has rare brain localization. Due to the infiltrative nature of the tumor and its sensitivity to radio and chemotherapy, particularly in patients with limited disease, we suggest that the complete surgical remove has a role for treatment in single and superficial lesions in immunocompetent patients. Moreover, stereotactic biopsy has been found helpful to determine the neoplasm diagnosis in deep lesions in non-immunocompetent patients. Recent guidelines published for immunocompetent patients with PCNSL recommended surgery for large, compressive lesions; however no consensus could be reached regarding the use of surgical resection for solitary, accessible lesions. ${ }^{35}$

\section{Conflicts of interest}

The authors declare that there are no potential conflicts of interest in relation to this study.

\section{Acknowledgements}

None. 


\section{References}

1. Abrey LE, Batchelor TT, Ferreri AJ, et al. Report of an internationa workshop to standardize baseline evaluation and response criteria for primary CNS lymphoma. J Clin Oncol. 2005;3:5034-5043.

2. Mead GM, Bleehen NM, Gregor A, et al. A medical research council randomized trial in patients with primary cerebral non-Hodgkin lymphoma: cerebral radiotherapy with and without cyclophosphamide, doxorubicin, vincristine, and prednisone chemotherapy. Cancer. 2000;89(6):1359-1370.

3. Bataille B, Delwail V, Menet E, et al. Primary intracerebral malignant lymphoma: report of 248 cases. J Neurosurg. 2000;92(2):261-266.

4. Brar R, Prasad A, Vermani N. Multifocal lateral and fourth ventricular B cell primary CNS lymphoma. Clin Neurol Neurosurg. 2012;114(3):281283.

5. GuhaThakurta N, Damek D, Pollack C, et al. Intravenous methotrexate as initial treatment for primary central nervous system lymphoma: Response to therapy and quality of life of patients. J Neurooncol. 1999;43(3):259 268.

6. BaraniskinA, Deckert M, Schulte-Altedorneburg G, et al. Current strategies in the diagnosis of diffuse large B-cell lymphoma of the central nervous system. Br J Haematol. 2012;156(4):421-432.

7. Brito $\mathrm{AB}$, Reis $\mathrm{F}$, de Souza $\mathrm{CA}$, et al. Intracranial primary dural diffuse large B-cell lymphoma successfully treated with chemotherapy. Int J Clin Exp Med. 2014;7(2):456-460.

8. Angelov L, Doolittle ND, Kraemer DF, et al. Blood-brain barrier disruption and intra-arterial methotrexate-based therapy for newly diagnosed primary CNS lymphoma: A multi-institutional experience. $J$ ClinOncol. 2009;27(21):3503-3509.

9. Wong ET, Tishler R, Barron L, et al. Immunochemotherapy with rituximab and temozolomide for central nervous system lymphomas. Cancer. 2004;101(1):139-45.

10. Zhang D, Hu LB, Henning TD, et al. MRI findings of primary CNS lymphoma in 26 immunocompetent patients. Korean $\mathrm{J}$ Radiol. 2010;11(3):269-277.

11. Donnarumma P, Pichierri A, Tarantino R, et al. 74 year-old woman with systemic lupus erythematosis and recent onset ataxia. Brain Pathol. 2014;24(2):193-194.

12. Ferreri AJ, Abrey LE, Blay JY, et al. Summary statement on primary central nervous system lymphomas from the Eighth International Conference on Malignant Lymphoma, Lugano, Switzerland, June 12 to 15, 2002. J Clin Oncol. 2003;21(12):2407-2714.

13. Elder JB, Chen TC. Surgical interventions for primary central nervous system lymphoma. Neurosurg Focus. 2006;21(5):E13.

14. Weller M, Martus P, Roth P, et al. Surgery for primary CNS lymphoma? Challenging a paradigm. NeuroOncol. 2012; 14(12):1481-1484.

15. Henry JM, Heffner RR, Dillard SH, et al. Primary malignant lymphomas of the central nervous system. Cancer. 1974;34(4):1293-1302.

16. De Angelis LM, Yahalom J, Heinemann MH, et al. Primary CNS lymphoma: combined treatment with chemotherapy and radiotherapy. Neurology. 1990;40(1):80-86.

17. Bellinzona M, Roser F, Ostertag H, et al. Surgical removal of primary central nervous system lymphomas (PCNSL) presenting as space occupying lesions: a series of 33 cases. Eur J Surg Oncol. 2005;31(1):100 105 .
18. Liu BL, Cheng JX, Zhang X, et al. Limited role of surgery in the management of primary central nervous system lymphoma (Review). Oncol Rep. 2009;22(3):439-449.

19. Jelicic J, Todorovic Balint M, Raicevic S, et al. The possible benefit from total tumour resection in primary diffuse large B-cell lymphoma of central nervous system - a onedecade single-centre experience. Br J Neurosurg. 2016;30(1):80-85.

20. Bierman PJ. Surgery for primary central nervous system lymphoma: is it time for reevaluation? Oncology (Williston Park). 2014;28(7):632-637.

21. Reni M, Ferreri AJ, Garancini MP, et al. Therapeutic management of primary central nervous system lymphoma in immunocompetent patients: results of a critical review of the. Ann Oncol. 1997;8(3):227-234.

22. Rubenstein JL, Hsi ED, Johnson JL, et al. Intensive chemotherapy and immunotherapy in patients with newly diagnosed primary CNS lymphoma: CALGB 50202 (Alliance50202). J Clin Oncol. 2013;31(25):3061-3068.

23. Dawei Chen, Weihong Gu, Wenzhong Li, et al. Primary diffuse large B-cell lymphoma of the central nervous system: A case report and literature review. Oncology letters. 2016;11(5):3085-3090.

24. Patrick LB, Mohile NA. Advances in primary central nervous system lymphoma. Curr Oncol Rep. 2015;17(12):60.

25. Ferreri AJ, Reni M, Pasini F, et al. A multicenter study of treatment of primary CNS lymphoma. Neurology. 2002;58(10):1513-1520.

26. Reni M, Ferreri AJ, Garancini MP, et al. Therapeutic management of primary central nervous system lymphoma in immunocompetent patients: results of a critical review of the literature. Ann Oncol. 1997;8(3):227234.

27. Mehmet B, Askin EH, Tulu A. Management of primary diffuse large b-cell lymphoma of the central nervous system: clinical study. $J$ of Neurological Sciences (Turkish). 2013;30:469-476.

28. Andres JM Ferreri. Trattamento dei linfomi primitivi e secondari del sistema nervoso centrale nei pazienti immunocompetenti. [haematologica reports]. 2006;2:8-11.

29. Glantz MJ, Cole BF, Recht L, et al. High-dose intravenous methotrexate for patients with non leukemic leptomeningeal cancer: Is intrathecal chemotherapy necessary? J Clin Oncol. 1998;16(4):1561-1567.

30. Yang Y, He J, Dang Yet al. One case report and literature review of primary central nervous system non Hodgkin's lymphoma. Lin Chuang Shen Jing Wai Ke Za Zh. 2014;5:373-375.

31. Barajas RF Jr, Rubenstein JL, Chang JS, et al. Diffusion-weighted MR imaging derived apparent diffusion coefficient is predictive of clinical outcome in primary central nervous system lymphoma. AJNR Am J Neuroradiol. 2010;31(1):60-66.

32. Yamanaka R, Morii K, Shinbo Y, et al. Results of treatment of 112 cases of primary CNS lymphoma. Jpn J Clin Oncol. 2008;38(5):373-380.

33. Weaver JD, Vinters HV, Koretz B, et al. Lymphomatosis cerebri presenting as rapidly progressive dementia. Neurologist. 2007;13(3):150-153.

34. Suh C, Kim JE and Yoon DH. Relapse pattern and prognostic factors for patients with primary CNS lymphoma. Korean J Hematol. 2012;47(2):155-156.

35. Hoang-Xuan K, Bessell E, Bromberg J, et al. Diagnosis and treatment of primary CNS lymphoma in immunocompetent patients: guidelines from the european association for neuro-oncology. Lancet Oncol. 2015;16(7):e322-332. 